International Mathematical Forum, Vol. 8, 2013, no. 2, 73 - 83

\title{
Nearring Ideals, Graphs and Cliques
}

\author{
Kedukodi Babushri Srinivas \\ Department of Mathematics \\ Manipal Institute of Technology, Manipal University \\ Manipal, Karnataka 576104, India \\ babushrisrinivas.k@manipal.edu, babushrisrinivas@yahoo.co.in \\ Kuncham Syam Prasad \\ Department of Mathematics \\ Manipal Institute of Technology, Manipal University \\ Manipal, Karnataka 576104, India. \\ syamprasad.k@manipal.edu,drkuncham@yahoo.com
}

Bh. Satyanarayana

Department of Mathematics

Acharya Nagarjuna University, Nagarjuna Nagar

Andhra Pradesh 522510, India

bhavanari2002@yahoo.co.in

\begin{abstract}
In this paper, we define the notion of fuzzy graph of a nearring $N$ with respect to a level ideal $\mu_{t}$ denoted by $(N, \mu, \sigma, t)$. The primary aim of this notion is to depict graphically the fuzzy character which is concealed algebraically in the examples of 3-prime fuzzy ideals of a nearring $N$. We find that if $N$ is a zero-symmetric nearring and $\mu$ is 3prime fuzzy ideal of $N$, then $(N, \mu, \sigma, t)$ has a special type of symmetry. We call this symmetry as the ideal symmetry of $(N, \mu, \sigma, t)$. We find the conditions under which the ideal symmetry of $(N, \mu, \sigma, t)$ implies $\mu$ is a 3-prime fuzzy ideal of $N$. Finally, we obtain a result which finds all the fuzzy cliques of $(N, \mu, \sigma, t)$ whenever $\mu$ is 3 -prime and $N$ is zerosymmetric.
\end{abstract}

Mathematics Subject Classification: 16Y30, 03E72, 16 Y99

Keywords: nearring, equiprime, 3-prime, c-prime, ideal, graph, clique 


\section{Introduction}

Yuan, Zhang and Ren [18] gave the definition of a fuzzy subgroup with a lower threshold $\alpha$ and an upper threshold $\beta$. Davvaz [8] used this idea to define the concept of a fuzzy ideal with thresholds $\alpha$ and $\beta$. Kedukodi, Kuncham and Bhavanari [13] used the concept of thresholds to define the notions of equiprime fuzzy ideal, 3-prime fuzzy ideal and c-prime fuzzy ideal of a nearring $N$. The primary benefit of the concept of thresholds is the choice for thresholds which gives rise to the fuzzy character in the examples.

In this paper, we define the notion of fuzzy graph of a nearring $N$ with respect to a level ideal. This notion depicts graphically the fuzzy character which is concealed algebraically in the examples of 3-prime fuzzy ideals of a nearring $N$. The idea comes from following facts:

(i) To depict fuzziness graphically, a natural mathematical tool is the fuzzy graph.

(ii) 3-prime fuzzy ideal of a nearring is characterized by its level ideals in the interval $(\alpha, \beta]$.

\section{Preliminary Notes}

We first summarize some basic definitions from fuzzy graph theory, most of which can be found in Mordeson and Nair [16].

Let $(V, E)$ be a graph with a vertex set $V$ and an edge set $E$ where $E \subseteq$ $V \times V$. A graph $(V, E)$ is said to be complete if $(u, v) \in E \forall u, v \in V, u \neq v$.

A clique of $(V, E)$ is a complete subgraph with maximum number of vertices. (Arora, Lund, Motwani, Sudan and Szegedy [3]) Computing a clique is not just NP-hard; it is hard even to approximate to any reasonable factor.

A fuzzy graph $H=(\mu, \sigma)$ of $(V, E)$ is defined by a fuzzy subset $\mu$ of $V$ and a fuzzy subset $\sigma$ of $E$ such that

$$
\sigma(x, y) \leq \mu(x) \wedge \mu(y) \forall x, y \in V .
$$

Let $t \in[0,1]$. The level subsets are defined as follows:

$$
\begin{gathered}
\mu_{t}=\{x \mid \mu(x) \geq t\} ; \\
\sigma_{t}=\{(x, y) \mid \sigma(x, y) \geq t\} .
\end{gathered}
$$

$\left(\mu_{t}, \sigma_{t}\right)$ is a graph with vertex set $\mu_{t}$ and edge set $\sigma_{t}$. 
A fuzzy graph $(\mu, \sigma)$ is said to be complete if

$$
\sigma(u, v)=\mu(u) \wedge \mu(v) \forall u, v \in V .
$$

A fuzzy clique of $(\mu, \sigma)$ is a complete subgraph with maximum number of vertices.

A standard reference on nearrings is Pilz [15] and on rings is Anderson and Fuller [2]. Throughout the paper, $N$ denotes a right nearring with finite cardinality. $Z_{n}$ denotes the ring of integers modulo $n$.

An ideal $I$ of $N$ is called prime if for any two ideals $A, B$ of $N, A B \subseteq I$ implies $A \subseteq I$ or $B \subseteq I$.

(Booth, Groenewald and Veldsman [5]) An ideal $I$ of $N$ is called equiprime if $a \in R \backslash I$ and $x, y \in R$ with ar $x-a r y \in I$ for all $r \in R$ implies $x-y \in I$. N is called an equiprime nearring if $\{0\}$ is an equiprime ideal of $N$. An equiprime nearring $N$ is zero-symmetric.

(Groenewald [11]) An ideal $I$ of $N$ is called 3-prime if $a, b \in N$ and $a r b \in I$ for all $r \in N$ implies $a \in I$ or $b \in I$.

(Groenewald [10]) An ideal $I$ of $N$ is called c-prime if $a, b \in N$ and $a b \in I$ implies $a \in I$ or $b \in I$.

(Veldsman [17] and Groenewald [10, 11]) The notions of equiprime ideal, 3prime ideal and prime ideal coincide in rings. In commutative rings the notions of equiprime ideal, 3-prime ideal, c-prime ideal and prime ideal coincide.

For a nonempty set $X$, the mapping $\mu: X \rightarrow[0,1]$ is called a fuzzy subset of $X$. (Davvaz [8]) Let $\alpha, \beta \in[0,1]$ and $\alpha<\beta$. Let $\mu$ be a fuzzy subset of a near-ring $\mathrm{N}$. Then $\mu$ is called a fuzzy ideal with thresholds of $\mathrm{N}$, if for all $x, y, i \in N$,

(i) $\alpha \vee \mu(x+y) \geq \beta \wedge \mu(x) \wedge \mu(y)$,

(ii) $\alpha \vee \mu(-x) \geq \beta \wedge \mu(x)$,

(iii) $\alpha \vee \mu(y+x-y) \geq \beta \wedge \mu(x)$,

(iv) $\alpha \vee \mu(x y) \geq \beta \wedge \mu(x)$,

(v) $\alpha \vee \mu(x(y+i)-x y) \geq \beta \wedge \mu(i)$.

We call $\alpha$ as the lower threshold of $N$ and $\beta$ as the upper threshold of $N$. Throughout this paper, $\alpha$ denotes the lower threshold of $N$ and $\beta$ denotes the upper threshold of $N$ and $\mu(0) \geq \beta$.

Theorem 2.1. (Davvaz [8]) Let $\mu$ be a fuzzy ideal of $N$. Then $\mu$ is a fuzzy ideal of $N$ if and only if for every $t \in(\alpha, \beta]$, the level subset $\mu_{t}$ is an ideal of $N$.

For the following definitions and results we refer to Kedukodi, Kuncham and Bhavanari $[12,13]$.

A fuzzy ideal $\mu$ of $N$ is called equiprime if for all $x, y, a \in N$,

$$
\alpha \vee \mu(a) \vee \mu(x-y) \geq \beta \wedge \inf _{r \in N} \mu(\operatorname{ar} x-\operatorname{ary}) .
$$


A fuzzy ideal $\mu$ of $N$ is called 3-prime if for all $a, b \in N$,

$$
\alpha \vee \mu(a) \vee \mu(b) \geq \beta \wedge \inf _{n \in N} \mu(a n b)
$$

A fuzzy ideal $\mu$ of $N$ is called $c$-prime if for all $a, b \in N$,

$$
\alpha \vee \mu(a) \vee \mu(b) \geq \beta \wedge \mu(a b)
$$

Theorem 2.2. Let $\mu$ be a fuzzy ideal of $N$. Then $\mu$ is an equiprime (resp. 3-prime, c-prime) fuzzy ideal of $N$ if and only if for every $t \in(\alpha, \beta]$, the level subset $\mu_{t}$ is an equiprime (resp. 3-prime, c-prime) ideal of $N$.

Theorem 2.3. Every equiprime fuzzy ideal of $N$ is a 3-prime fuzzy ideal of $N$.

Theorem 2.4. Every c-prime fuzzy ideal of $N$ is a 3-prime fuzzy ideal of $N$.

Theorem 2.5. Let $N$ be a commutative ring. Then the following are equivalent:

(i) $\mu$ is an equiprime fuzzy ideal of $N$.

(ii) $\mu$ is a 3-prime fuzzy ideal of $N$.

(iii) $\mu$ is a c-prime fuzzy ideal of $N$.

\section{$3 \quad$ Fuzzy Graph of a Nearring with respect to a Level Ideal}

Definition 3.1. Let $\mu: N \rightarrow(0,1]$ be a fuzzy ideal of $N$ with thresholds $\alpha$ and $\beta$. Let $t \in(\alpha, \beta]$ be fixed.

Define $\sigma: N \times N \rightarrow[0,1]$ as follows:

$$
\sigma(x, y)= \begin{cases}\mu(x) \wedge \mu(y) & x \neq y \text { and }\left(x N y \subseteq \mu_{t} \text { or } y N x \subseteq \mu_{t}\right) \\ 0 & \text { Otherwise }\end{cases}
$$

Then the fuzzy graph $(N, \mu, \sigma)$ is called the fuzzy graph of $N$ with respect to the level ideal $\mu_{t}$. We denote the fuzzy graph of $N$ with respect to the level ideal $\mu_{t}$ by $(N, \mu, \sigma, t)$. 
Remark 3.2. Suppose $N$ is a commutative ring with unit element 1. Let $\mu: N \rightarrow(0,1]$ be a fuzzy ideal of $N$ and $\mu_{t}$ be any level ideal of $N$. Then the condition $\left(x N y \subseteq \mu_{t}\right.$ or $\left.y N x \subseteq \mu_{t}\right)$ is equivalent to $x y \in \mu_{t}$. Also, the condition $x N x \subseteq \mu_{t}$ is equivalent to $x^{2} \in \mu_{t}$.

Proof. Suppose $x N y \subseteq \mu_{t}$. This implies $x \cdot 1 \cdot y \in \mu_{t}$. Hence $x y \in \mu_{t}$. If $x=y$ then $x^{2} \in \mu_{t}$. Conversely, let $x y \in \mu_{t}$. As $\mu_{t}$ is an ideal of the ring $N$, we have $x y N \subseteq \mu_{t}$. As $N$ is commutative, $\left(x N y \subseteq \mu_{t}\right.$ or $\left.y N x \subseteq \mu_{t}\right)$. If $x=y$ then $x N x \subseteq \mu_{t}$.

In this sequel, fuzzy graphs will have the following colour conventions:

(i) Red colour for the elements of $\mu_{t}$.

(ii) Red colour for the edges between any two elements of $\mu_{t}$.

(iii) Blue colour for the elements of $N \backslash \mu_{t}$.

(iv) Olive colour for the edges between the elements of $\mu_{t}$ and $N \backslash \mu_{t}$.

Example 3.3. Consider $N=Z_{12}=\{0,1,2, \ldots, 11\}$ (Ring of integers modulo 12). $\quad N=Z_{12}$ is a commutative ring with unit element 1 . Let $a, b \in$ $(0.2,0.8)$ and $a \neq b$. Define a fuzzy subset $\mu: Z_{12} \rightarrow[0,1]$ by

$$
\mu(x)= \begin{cases}0.9 & \text { if } x=6 \\ 0.8 & \text { if } x=0 \\ a & \text { if } x \in\{3,9\} \\ b & \text { if } x \in\{2,4,8,10\} \\ 0.2 & \text { elsewhere }\end{cases}
$$

Take thresholds $\alpha=a \wedge b$ and $\beta=a \vee b$. Then $\mu$ is a fuzzy ideal of $Z_{12}$. Let $t=\beta$. To get a sketch of $(N, \mu, \sigma, \beta)$, we choose some values of $a$ and $b$.

Choose $a=0.6$ and $b=0.4$. Then $\alpha=0.4$ and $\beta=0.6$. We have $t=\beta=0.6$ and $\mu_{t}=\{0,3,6,9\}$. We form a table for $\sigma$ as follows:

\begin{tabular}{|c|c|c|c|c|c|c|c|c|c|c|c|c|}
\hline$\sigma(x, y)$ & $y=0$ & $y=1$ & $y=2$ & $y=3$ & $y=4$ & $y=5$ & $y=6$ & $y=7$ & $y=8$ & $y=9$ & $y=10$ & $y=11$ \\
\hline$x=0$ & 0 & 0.2 & 0.4 & 0.6 & 0.4 & 0.2 & 0.8 & 0.2 & 0.4 & 0.6 & 0.4 & 0.2 \\
\hline$x=1$ & 0.2 & 0 & 0 & 0.2 & 0 & 0 & 0.2 & 0 & 0 & 0.2 & 0 & 0 \\
\hline$x=2$ & 0.4 & 0 & 0 & 0.4 & 0 & 0 & 0.4 & 0 & 0 & 0.4 & 0 & 0 \\
\hline$x=3$ & 0.6 & 0.2 & 0.4 & 0 & 0.4 & 0.2 & 0.6 & 0.2 & 0.4 & 0.6 & 0.4 & 0.2 \\
\hline$x=4$ & 0.4 & 0 & 0 & 0.4 & 0 & 0 & 0.4 & 0 & 0 & 0.4 & 0 & 0 \\
\hline$x=5$ & 0.2 & 0 & 0 & 0.2 & 0 & 0 & 0.2 & 0 & 0 & 0.2 & 0 & 0 \\
\hline$x=6$ & 0.8 & 0.2 & 0.4 & 0.6 & 0.4 & 0.2 & 0 & 0.2 & 0.4 & 0.6 & 0.4 & 0.2 \\
\hline$x=7$ & 0.2 & 0 & 0 & 0.2 & 0 & 0 & 0.2 & 0 & 0 & 0.2 & 0 & 0 \\
\hline$x=8$ & 0.4 & 0 & 0 & 0.4 & 0 & 0 & 0.4 & 0 & 0 & 0.4 & 0 & 0 \\
\hline$x=9$ & 0.6 & 0.2 & 0.4 & 0.6 & 0.4 & 0.2 & 0.6 & 0.2 & 0.4 & 0 & 0.4 & 0.2 \\
\hline$x=10$ & 0.4 & 0 & 0 & 0.4 & 0 & 0 & 0.4 & 0 & 0 & 0.4 & 0 & 0 \\
\hline$x=11$ & 0.2 & 0 & 0 & 0.2 & 0 & 0 & 0.2 & 0 & 0 & 0.2 & 0 & 0 \\
\hline $\operatorname{deg}(y)$ & 4.4 & 0.8 & 1.6 & 4.2 & 1.6 & 0.8 & 4.4 & 0.8 & 1.6 & 4.2 & 1.6 & 0.8 \\
\hline
\end{tabular}




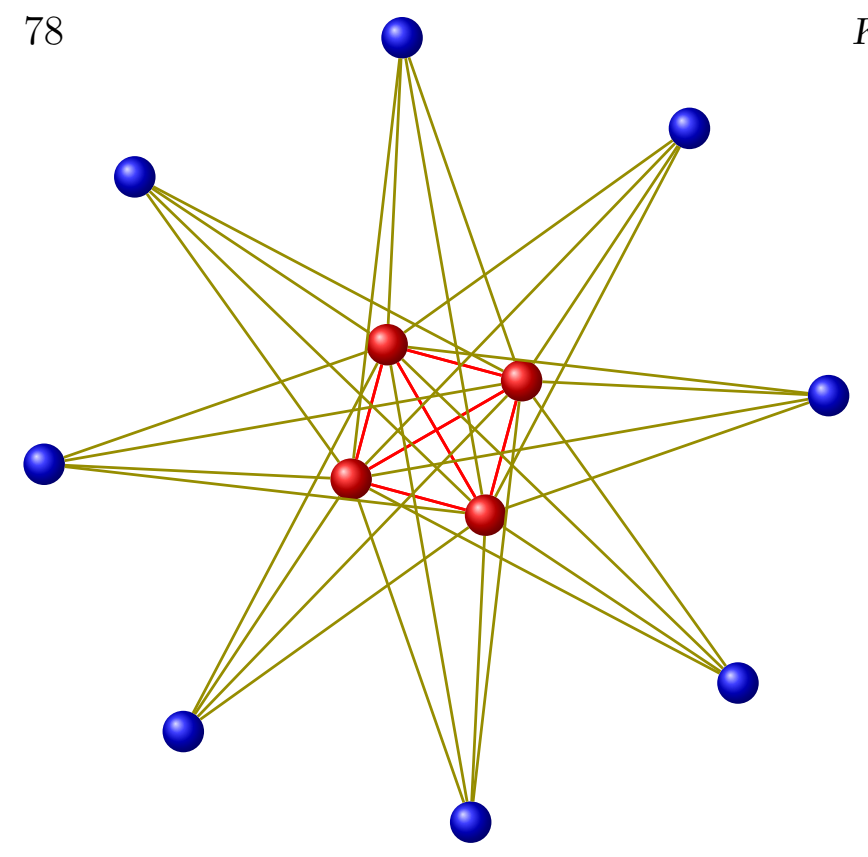

Kedukodi Babushri Srinivas et al

Figure 1: Graph when $a>b$.

The graph is given in Figure 1.

Now we choose $a=0.4$ and $b=0.6$. Then $\alpha=0.4$ and $\beta=0.6$. We have $t=\beta=0.6$ and $\mu_{t}=\{0,2,4,6,8,10\}$. The table for $\sigma$ is given below and graph is depicted in Figure 2.

\begin{tabular}{|c|c|c|c|c|c|c|c|c|c|c|c|c|}
\hline $\bar{\sigma}(x, y)$ & $y=0$ & $y=1$ & $y=2$ & $y=3$ & $y=4$ & $y=5$ & $y=6$ & $y=7$ & $y=8$ & $y=9$ & $y=10$ & $y=11$ \\
\hline$x=0$ & 0 & 0.2 & 0.6 & 0.4 & 0.6 & 0.2 & 0.8 & 0.2 & 0.6 & 0.4 & 0.6 & 0.2 \\
\hline$x=1$ & 0.2 & 0 & 0.2 & 0 & 0.2 & 0 & 0.2 & 0 & 0.2 & 0 & 0.2 & 0 \\
\hline$x=2$ & 0.6 & 0.2 & 0 & 0.4 & 0.6 & 0.2 & 0.6 & 0.2 & 0.6 & 0.4 & 0.6 & 0.2 \\
\hline$x=3$ & 0.4 & 0 & 0.4 & 0 & 0.4 & 0 & 0.4 & 0 & 0.4 & 0 & 0.4 & 0 \\
\hline$x=4$ & 0.6 & 0.2 & 0.6 & 0.4 & 0 & 0.2 & 0.6 & 0.2 & 0.6 & 0.4 & 0.6 & 0.2 \\
\hline$x=5$ & 0.2 & 0 & 0.2 & 0 & 0.2 & 0 & 0.2 & 0 & 0.2 & 0 & 0.2 & 0 \\
\hline$x=6$ & 0.8 & 0.2 & 0.6 & 0.4 & 0.6 & 0.2 & 0 & 0.2 & 0.6 & 0.4 & 0.6 & 0.2 \\
\hline$x=7$ & 0.2 & 0 & 0.2 & 0 & 0.2 & 0 & 0.2 & 0 & 0.2 & 0 & 0.2 & 0 \\
\hline$x=8$ & 0.6 & 0.2 & 0.6 & 0.4 & 0.6 & 0.2 & 0.6 & 0.2 & 0 & 0.4 & 0.6 & 0.2 \\
\hline$x=9$ & 0.4 & 0 & 0.4 & 0 & 0.4 & 0 & 0.4 & 0 & 0.4 & 0 & 0.4 & 0 \\
\hline$x=10$ & 0.6 & 0.2 & 0.6 & 0.4 & 0.6 & 0.2 & 0.6 & 0.2 & 0.6 & 0.4 & 0 & 0.2 \\
\hline$x=11$ & 0.2 & 0 & 0.2 & 0 & 0.2 & 0 & 0.2 & 0 & 0.2 & 0 & 0.2 & 0 \\
\hline $\operatorname{deg}(y)$ & 4.8 & 1.2 & 4.6 & 2.4 & 4.6 & 1.2 & 4.8 & 1.2 & 4.6 & 2.4 & 4.6 & 1.2 \\
\hline
\end{tabular}

Definition 3.4. The fuzzy graph $(N, \mu, \sigma, t)$ is called ideal symmetric if for every pair of vertices $x, y$ in $(N, \mu, \sigma, t)$ with an edge between them, either $[\sigma(x, z)>0 \forall z \neq x ; z \in N]$ or $[\sigma(y, z)>0 \forall z \neq y ; z \in N]$.

Remark 3.5. An element $x$ is connected to every other element in $(N, \mu, \sigma, t)$ if and only if $\sigma(x, z)>0 \forall z \neq x ; z \in N$. 


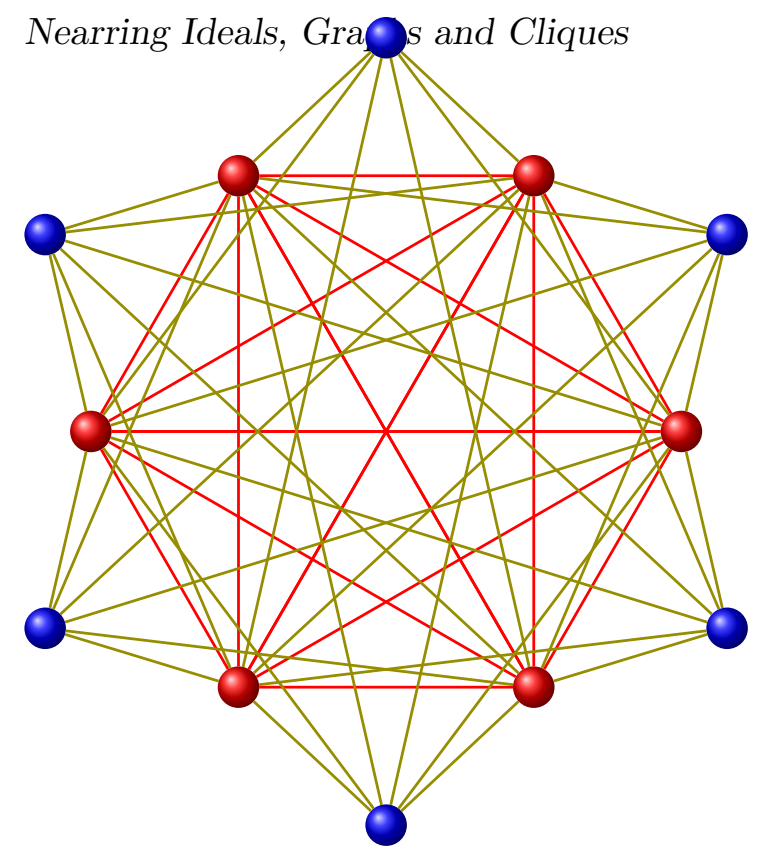

Figure 2: Graph when $a<b$.

Lemma 3.6. (i) Let $\mu$ be a 3-prime fuzzy ideal of $N$ and $x$ be a vertex in $(N, \mu, \sigma, t)$. If $\sigma(x, z)>0 \forall z \neq x ; z \in N$ then $x \in \mu_{t}$.

(ii) Let $N$ be a zero-symmetric nearring and $x$ be a vertex in $(N, \mu, \sigma, t)$. If $x \in \mu_{t}$ then $\sigma(x, z)>0 \quad \forall z \neq x ; z \in N$.

(iii) Let $N$ be a zero-symmetric nearring. Then $\mu$ is 3-prime fuzzy ideal of $N$ if and only if every element $x \in \mu_{t}$ is connected to all other elements of $N$ in $(N, \mu, \sigma, t)$.

(iv) Let $N$ be a zero-symmetric nearring. If $\mu$ is 3-prime fuzzy ideal $N$ then $\left(\mu_{t}, \mu, \sigma, t\right)$ is complete subgraph of $(N, \mu, \sigma, t)$.

Proof. To prove (i), let $x$ be a vertex in $(N, \mu, \sigma, t)$ and assume $x$ is connected to every other element of $N$. Then $x N y \subseteq \mu_{t}$ or $y N x \subseteq \mu_{t}$ for all $y \in N$ such that $y \neq x$. Without loss of generality, assume $x N y \subseteq \mu_{t}$ for all $y \in N$ such that $y \neq x$. If $\mu_{t}=N$ then $x \in \mu_{t}$. Let $\mu_{t} \neq N$. Choose $y \in N \backslash \mu_{t}$. As $\mu_{t}$ is a 3-prime ideal of $N$ and $x N y \subseteq \mu_{t}$, we get $x \in \mu_{t}$. To prove (ii), let $x \in \mu_{t}$. If $x=0$ then the result is true. Let $x \neq 0$. If possible, suppose that there exists a vertex $y$ such that $y$ is not connected to $x$ in $(N, \mu, \sigma, t)$. This implies $x N y \nsubseteq \mu_{t}$ and $y N x \nsubseteq \mu_{t}$. Now as $x \in \mu_{t}$ and $\mu_{t}$ is an ideal of $N$, we have $x N \subseteq \mu_{t}$. Hence $x N y \subseteq\left(\mu_{t}\right) y$. But since $N$ is zero-symmetric, we have $\left(\mu_{t}\right) y \subseteq \mu_{t}$. Thus we get $x N y \subseteq \mu_{t}$, a contradiction. This proves $x$ is connected 
to every other element of $N$ in $(N, \mu, \sigma, t)$. The result (iii) follows from (i) and (ii). The result (iv) follows from (iii).

Theorem 3.7. Let $\mu$ be a fuzzy ideal of $N$.

(a) Suppose

(i) $N$ is zero-symmetric;

(ii) $\mu$ is 3-prime.

Then $(N, \mu, \sigma, t)$ is ideal symmetric for all $t \in(\alpha, \beta]$.

(b) Suppose

(i) $(N, \mu, \sigma, t)$ is ideal symmetric for all $t \in(\alpha, \beta]$;

(ii) For every $x \in N$ and for every $t \in(\alpha, \beta], x N x \subseteq \mu_{t}$ implies $x \in \mu_{t}$;

(iii) For every $x \in N$ and for every $t \in(\alpha, \beta], x$ is connected to every other element of $N$ in $(N, \mu, \sigma, t)$ implies $x \in \mu_{t}$.

Then $\mu$ is 3-prime.

Proof. To prove $(a)$, let $t \in(\alpha, \beta]$. Let $x, y$ be distinct vertices of $(N, \mu, \sigma, t)$ with an edge between $x$ and $y$. Then $x N y \subseteq \mu_{t}$ or $y N x \subseteq \mu_{t}$. Without loss of generality, assume $x N y \subseteq \mu_{t}$. As $\mu_{t}$ is 3-prime ideal of $N$, we have $x \in \mu_{t}$ or $y \in \mu_{t}$. Now as $N$ is a zero-symmetric nearring, by Lemma 3.6 (ii) we conclude that $(N, \mu, \sigma, t)$ is ideal symmetric. To prove (b), let $t \in(\alpha, \beta]$ and $x, y \in N$ and $x N y \subseteq \mu_{t}$. If $\mu_{t}=N$ then $x \in \mu_{t}$. Hence assume $\mu_{t} \neq N$. If $x=y$ then $x \in I$. Let $x \neq y$. Now there exists an edge between $x$ and $y$ in $(N, \mu, \sigma, t)$. As $(N, \mu, \sigma, t)$ is ideal symmetric, either $x$ is connected to every other element of $N$ in $(N, \mu, \sigma, t)$ or $y$ is connected to every other element of $N$ in $(N, \mu, \sigma, t)$. By Lemma 3.6 (i), we get $x \in \mu_{t}$ or $y \in \mu_{t}$. Thus $\mu$ is a 3 -prime fuzzy ideal of $N$.

Remark 3.8. The fuzzy ideal defined in the Example 3.3 is a 3-prime fuzzy ideals of a zero-symmetric nearring. The fuzzy graphs shown in Fig 1, Fig 2 are ideal symmetric.

Example 3.9. Let $N$ be an equiprime nearring. Then $N$ is zero-symmetric. Take $a, b \in[0,1]$ such that $a<b$. Define a fuzzy subset $\mu$ by

$$
\mu(x)= \begin{cases}b & \text { if } x=0 \\ a & \text { otherwise }\end{cases}
$$


Choose thresholds $\alpha=a$ and $\beta=b$. Then $\mu$ is an equiprime fuzzy ideal of $N$. This implies $\mu$ is a 3-prime fuzzy ideal of $N$. By Theorem 3.7, $(N, \mu, \sigma, t)$ is ideal symmetric for all $t \in(a, b]$.

Remark 3.10. The class of equiprime nearrings is well-known in nearrings. An extensive set of examples of equiprime nearrings can be found in Veldsman [17].

Corollary 3.11. Suppose $N$ is a commutative ring with unit element 1. Let $\mu$ be a fuzzy ideal of $N$. Suppose for every $x \in N$ and for every $t \in(\alpha, \beta]$, $x^{2} \in \mu_{t}$ implies $x \in \mu_{t}$. If for every $t \in(\alpha, \beta],(N, \mu, \sigma, t)$ is ideal symmetric then $\mu$ is a c-prime fuzzy ideal of $N$.

Proof. Let $t \in(\alpha, \beta]$. By Remark 3.2, the condition $x^{2} \in \mu_{t}$ is equivalent to the condition $x N x \subseteq \mu_{t}$. Now, let $x \in N$ such that $\sigma(x, z)>0 \forall z \neq x ; z \in N$. If $x=0$ then $x \in \mu_{t}$. If $\mu_{t}=N$ then $x \in \mu_{t}$. Hence assume $x \neq 0$ and $\mu_{t} \neq N$. We have $x N y \subseteq \mu_{t}$ and $y N x \subseteq \mu_{t}$ for all $y \in N$ such that $y \neq x$. If $x=1$ then $1 \cdot 1 \cdot y \in \mu_{t}$ for all $y \in N$ such that $y \neq 1$. Since $\mu_{t}$ is an ideal of $N, N$ must have exactly two elements namely 0 and the unit element 1 . Now, $Z_{2}$ is the only ring of order 2 with unit element 1 . Note that $\mu_{t}=\{0\}$ is a c-prime ideal of $Z_{2}$. Suppose $x \neq 1$. Then we have $x \cdot 1 \cdot 1 \in \mu_{t}$. Hence we get $x \in \mu_{t}$. Using the part (b) of the Theorem 3.7, $\mu_{t}$ is 3 -prime. This implies $\mu$ is 3 -prime. In a commutative ring, the concepts of 3-prime fuzzy ideal and c-prime fuzzy ideal coincide. Hence $\mu$ is a c-prime fuzzy ideal of $N$.

Theorem 3.12. Let $\mu$ be a 3-prime fuzzy ideal of a zero-symmetric nearring $N$. Consider $(N, \mu, \sigma, t)$ and $a \notin \mu_{t}$. Then $C=\mu_{t} \cup\{a\}$ is a fuzzy clique of $(N, \mu, \sigma, t)$.

Proof. By Lemma 3.6 (iv), $\left(\mu_{t}, \mu, \sigma, t\right)$ is complete subgraph of $(N, \mu, \sigma, t)$. Let $a \notin \mu_{t}$ and $C=\mu_{t} \cup\{a\}$. By Lemma 3.6 (iii), every element of $\mu_{t}$ is connected to $a$. This proves that $(C, \mu, \sigma, t)$ is complete. Now we will prove that $C$ is maximal. First, we show that if $x, y \in N \backslash \mu_{t}$ and $x \neq y$ then $x$ is not connected to $y$ in $(N, \mu, \sigma, t)$. If possible suppose $x$ is connected to $y$. Then $x N y \subseteq \mu_{t}$ or $y N x \subseteq \mu_{t}$. Without loss of generality, assume $x N y \subseteq \mu_{t}$. As $\mu_{t}$ is 3-prime ideal of $N$, we get $x \in \mu_{t}$ or $y \in \mu_{t}$. This is a contradiction. Now, let $C_{1}=C \cup\{b\}$; $b \notin \mu_{t}$ and $b \neq a$. Then $\sigma(a, b)=0 \neq \mu(a) \wedge \mu(b)$. This implies $\left(C_{1}, \mu, \sigma, t\right)$ is not complete. Hence $\left(C_{1}, \mu, \sigma, t\right)$ cannot be a fuzzy clique. Thus $C=\mu_{t} \cup\{a\}$ is a fuzzy clique of $(N, \mu, \sigma, t)$.

Remark 3.13. Consider $(N, \mu, \sigma, t)$ defined in Example 3.3. A fuzzy clique of $(N, \mu, \sigma, t)$ can be any one of the networks shown in Figure 3 or Figure 4 (Note that the fuzzy clique depends on the values of a and $b$ in Example 3.3). 


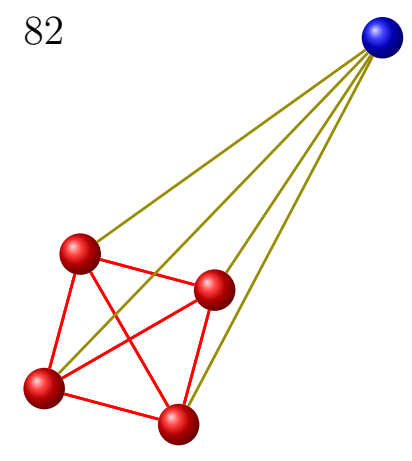

Kedukodi Babushri Srinivas et al

Figure 3: Example 3.3, a clique when $a>b$.

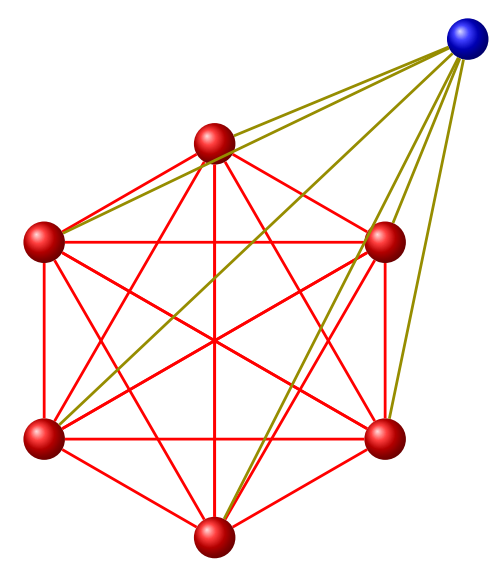

Figure 4: Example 3.3, a clique when $a<b$.

ACKNOWLEDGEMENTS. The first and the second authors acknowledge Manipal University and the third author acknowledges Acharya Nagarjuna University for their encouragement.

\section{References}

[1] S. Abou-Zaid, On fuzzy subnear-rings and ideals, Fuzzy Sets and Systems, 44 (1991) 139-146.

[2] F. W. Anderson, K. R. Fuller, Rings and Categories of Modules, Second edition, Springer-Verlag, USA, 1992.

[3] A. Arora, C. Lund, R. Motwani, M. Sudan and M. Szegedy, Proof verification and the intractability of approximation problems, IEEE Symposium on Foundations of Computer Science, (1992) 13-22. 
[4] N. L. Biggs, Discrete Mathematics, Second edition, Oxford University Press, 2003.

[5] G. L. Booth, N. J. Groenewald and S Veldsman, A Kurosh-Amitsur prime radical for near-rings, Comm. Algebra, 18 (9) (1990) 3111-3122.

[6] S. Bhavanari, S.P. Kuncham, B.S. Kedukodi, Graph of a nearring with respect to an ideal, Comm. Algebra, 38 (5) (2010) 1957-1967.

[7] S. K. Bhakat and P. Das, Fuzzy subrings and ideals redefined, Fuzzy Sets and Systems, 81 (1996) 383-393.

[8] B. Davvaz, $(\epsilon, \epsilon \vee q)$-fuzzy subnear-rings and ideals, Soft Comput., 10 (2006) 206-211.

[9] N. Graham and F. Harary, Some small sized spanning subgraphs of a hypercube, Computers Math. Applic., 34 (11)(1997) 51-57.

[10] N. J. Groenewald, The completely prime radical in nearrings, Acta Math. Hung., 51 (3-4)(1988) 301-305.

[11] N. J. Groenewald, Different prime ideals in near-rings, Comm. Algebra, 19 (1991) 2667-2675.

[12] B. S. Kedukodi, S. Bhavanari and S. P. Kuncham, C-prime fuzzy ideals of nearrings, Soochow J. Math., 33 (4) (2007) 891-901.

[13] B. S. Kedukodi, S. P. Kuncham and S. Bhavanari, Equiprime, 3-prime and c-prime fuzzy ideals of nearrings, Soft Comput., 13 (2009) 933-944.

[14] B. S. Kedukodi, S. P. Kuncham and S. Bhavanari, Reference points and roughness, Inform. Sci., 180 (2010) 3348-3361.

[15] G. Pilz, Near-Rings, Revised edition, North Holland, 1983.

[16] J. N. Mordeson, P. S. Nair, Fuzzy Graphs and Fuzzy Hypergraphs, PhysicaVerlag, Heidelberg, 2000.

[17] S. Veldsman, On equiprime near-rings, Comm. Algebra, 20 (9) (1992) 2569-2587.

[18] X. Yuan, C. Zhang and Y. Ren, Generalized fuzzy groups and manyvalued implications, Fuzzy Sets and Systems, 138 (2003) 205-211.

Received: September, 2012 